\title{
Smoking During Pregnancy and Smoking Cessation Services
}

\author{
Linda Bauld \\ University of Bath, United Kingdom
}

\begin{abstract}
$T^{10}$ 2008 UK National Smoking Cessation Conference (UKNSSC) included a number of oral and poster presentations on the theme of smoking during pregnancy. This is a challenging area of research and practice and one in which new evidence - both about the effects of smoking in pregnancy and about smoking cessation interventions - is regularly emerging. Papers at UKNSCC explored why few women access support to stop (Felix Naughton), how best to refer women to specialist services (Joan Braithwaite), social marketing approaches (Deborah Richardson and Wendy Dudley) and physical activity for smoking cessation during pregnancy (Michael Ussher). The conference opened with a plenary presentation that explored the extent of smoking during pregnancy and women's accounts of quit attempts, cessation and relapse. It also examined what more could be done to improve access to stop smoking services for pregnant women and increase the proportion of women who quit. This article reviews some of the evidence presented at UKNSSC, focusing in particular on the need for improved identification, referral, engagement and treatment of pregnant smokers.
\end{abstract}

\section{Background}

Obtaining accurate figures on the extent of smoking during pregnancy is a challenge in many countries, and the United Kingdom is no exception. Prevalence figures in the United Kingdom are drawn from the infant feeding survey, which is conducted infrequently (every 5 years) and relies on self-report data collected from women following delivery (Information Centre, 2007). The survey asks two main questions about smoking whether women have smoked in the 12 months before or during pregnancy, and whether they have smoked throughout pregnancy.

Findings from the most recent survey (2005) show that smoking rates throughout pregnancy varied across the UK from 22\% in Wales to 20\% in Scotland and 18\% in Northern Ireland, with the rate for England and the UK overall at $17 \%$. This represents a decline from the UK average of $20 \%$ recorded by the same survey in 2000 . However, smoking rates differ significantly depending on the age and socio-economic status of the pregnant woman. For example, pregnant mothers who are 20 or under are more than three times as likely to smoke before or during pregnancy as mothers aged 35 or over.
In addition, mothers in routine and manual occupations are more than four times as likely as those in managerial and professional occupations to have smoked throughout pregnancy (29\% and $7 \%$ respectively). What is of particular concern is that comparisons between 2000 and 2005 by socio-economic group in England show a slight increase in smoking throughout pregnancy for routine and manual groups, suggesting that inequalities in this area may be increasing rather than reducing (Information Centre, 2007).

Governments have used the infant feeding survey figures as the basis for targets to reduce smoking during pregnancy, particularly in England where the main target is to reduce the rate of smoking during pregnancy from an average of $23 \%$ in 1998 to an average of $15 \%$ by 2010 .

Targets vary in other parts of the United Kingdom but at a local level additional requirements have been placed on the health care system (particularly primary care trusts in England) to reduce the proportion of mothers smoking at the time of delivery (data collected through hospital maternity records) by $1 \%$ each year for three years up to 2010 (O'Gorman, 2008).

These targets reflect government awareness of the adverse effects of smoking during pregnancy that

Address for correspondence: Linda Bauld, Reader in Social Policy, UK Centre for Tobacco Control Studies, Dept of Social and Policy Sciences, University of Bath, Bath, BA2 7AY, United Kingdom. E-mail: L.Bauld@bath.ac.uk 
include 4,000 deaths per year in the United Kingdom from miscarriage and stillbirth, more preterm and low birth weight babies, an increase in sudden infant death, asthma and attention deficit hyperactivity disorder (RCP, 1992, Charlton, 1996, Bastra et al., 2003). Increasingly they also reflect an understanding by policymakers that reducing smoking during pregnancy is necessary to protect children from exposure to the damaging effects of second hand smoke in the home and, in turn, to reduce the risk that the children will follow in the footsteps of their smoking parents and become smokers themselves (BMA, 2007).

\section{Identification}

Despite the existence of targets to reduce smoking during pregnancy in the United Kingdom and some evidence of progress towards meeting those targets, research suggests that problems remain. The majority of pregnant women who quit smoking (between 9-45\%) do so without any formal intervention (Lawrence et al., 2005; Greaves et al. 2003). These quitters tend to be older, less addicted, more highly educated, and less likely to have a partner who smokes (Greaves et al., 2003). Women who are unable to quit on their own (most commonly on or around the confirmation of their pregnancy) face greater challenges in trying to stop. These women would benefit from accessing formal support, of the kind offered by NHS stop smoking services in the UK, but few do so. One recent study estimated that 17\% of pregnant smokers in England set a quit date with NHS stop smoking services in 2007 (Naughton, 2008). The figure is, however, likely to be lower than this in many parts of the country and national monitoring data in Scotland suggests that just 10\% of pregnant smokers there engage with services (ISD, 2008).

There are a number of explanations for this. Some of these are specific to pregnancy, while others are common to most smokers trying to quit. Previous research has shown that pregnant women face multiple barriers in attempting to quit smoking including lack of understanding about the risks, fear of failure, fear of weight gain, factors related to addiction and withdrawal and factors related to their wider social context (Greaves et al., 2003). One recent study explored why women in the United States and the United Kingdom did not choose to access smoking cessation services and found that unwillingness to seek help and lack of available local services may also play a role (Ussher et al., 2006). Another study (Lowry et al., 2004) focusing on the north east of England found that unsatisfactory information, lack of enthusiasm or empathy from healthcare professionals and short-term support, all contributed to few women accessing services.

However, one of the main barriers to increasing uptake of smoking cessation services is a lack of reliable information on the real extent of smoking during pregnancy. The survey figures outlined above are likely to underestimate the number of women who continue to smoke when pregnant, and without being able to accurately identify the size of the problem, it is difficult for services to respond appropriately.

Studies in the United Kingdom and internationally have shown that women have a tendency to underreport smoking during pregnancy. One study conducted in New Zealand in the 1990s compared self-report data on smoking during pregnancy with serum cotinine verified data from routine pregnancy blood samples (Ford et al., 1997). A more recent study in Glasgow compared selfreport data with the results of routine $\mathrm{CO}$ monitoring and found a similar mismatch (in $24 \%$ of cases) between self-report and validated smoking status (Usmani et al., 2008). Routine validation of the smoking status of pregnant women is required to overcome the problem of underreporting. In some parts of the United Kingdom, $\mathrm{CO}$ monitoring during maternity booking is now in place and this represents progress. However, screening of routine pregnancy blood samples for cotinine is a more accurate alternative and a debate about the feasibility and acceptability of its introduction is needed (ASH, 2008).

An additional barrier to identifying the number of women smoking during pregnancy in the United Kingdom relates to problems with the recording of smoking status at maternity booking. Studies have shown the some midwives are still reluctant to ask women about their smoking status. In some cases even when the question is asked, the response is not recorded. The result is that smoking status is recorded as 'unknown' in 5\% of cases in Scotland and up to 6\% in England according to maternity records (ISD, 2008, O'Gorman, 2008). A recent study in Scotland found that under-recording was more common when the woman was from a deprived area suggesting that we may currently be missing an opportunity to address smoking during pregnancy with women from communities where smoking rates are highest (Bauld et al., 2007).

\section{Referral and Engagement}

Guidelines for health professionals in the United Kingdom and a number of other countries specify that once tobacco use during pregnancy has been identified, women should be given brief advice to stop and ideally be referred on for further support, whether that be to telephone quit lines for pregnant women or, in the case of the United Kingdom, intensive support of the kind provided by NHS stop smoking services. However, the gap between identification of smoking and referral to cessation services can be significant. A recent national audit of smoking cessation services for pregnant women in Scotland (Macaskill et al., 2008) found that the proportion of self-reported smokers referred to services by midwives varied from $16 \%$ to $93 \%$ between maternity hospitals. The hospital with the high referral rate had 
routine $\mathrm{CO}$ monitoring in place for all women and operated an'opt out' process where the details of all smokers, except in exceptional circumstances, were passed to specialist smoking cessation staff.

The audit found that engagement rates (ie. the proportion of self-reported smokers who then attended an appointment with a cessation specialist) were higher in the unit with the opt-out policy in place (17\% of selfreported smokers attended an appointment in this hospital compared with, for instance, $8 \%$ in units with opt-in policies) (Macaskill et al., 2008). Even though overall engagement rates remained low, this finding suggests that referring on all identified smokers may result in more coming into contact with specialist services, and in turn enhancing the possibility that these women may be successful in a quit attempt.

\section{Treatment}

Smoking cessation interventions with pregnant women can be effective, and routine data from England shows that in the period between 1999 and 2007, between 35 to $51 \%$ of pregnant women who engaged with NHS stop smoking services and set a quit date reported quitting by at 4 weeks (Bell et al., 2007). In Scotland, 4 -week quit rates for pregnant women have not been reported in national statistical bulletins, but findings from the audit mentioned above provide some figures for Glasgow, Scotland's largest city. Four week quit rates for women treated by a specialist smoking cessation adviser in the three maternity hospitals in the city from May 2005 to April 2006 were $34 \%, 33 \%$ and $50 \%$ respectively (Macaksill et al., 2008).

Yet short-term outcomes from treatment tell us little about two things. The first is how the proportion of women quitting with support from cessation services compares with the population of identified smokers - in other words, what proportion of women smoking during pregnancy are helped to stop by specialist services. The second is to what extent short term quit rates translate into abstinence up to delivery and post-partum.

Even though monitoring data from NHS stop smoking services suggests that a significant proportion of pregnant women who set a quit date with a service do stop by 4 weeks, they still represent a tiny proportion of the population of women smoking during pregnancy in the United Kingdom. The problems outlined above in relation to identifying pregnant smokers, referring them to services and encouraging engagement with services combine with relapse during treatment to result in low success rates. Findings from the analysis of data from Glasgow hospitals described above suggests that the number of women quitting at four weeks as a proportion of all identified smokers that year ranged from $5.4 \%$ in the maternity unit with the 'opt-out' referral policy to $5 \%$ and $3.4 \%$ in the two other hospitals (Macaskill et al., 2008). What this suggests is that the fundamental problem is not so much with treatment efficacy but with reach. Clearly CO monitoring and optout referral systems can improve reach, and this is one reason why more maternity units are now adopting these procedures. However, other factors that can encourage women to access support are adequate training for GPs and midwives in giving brief advice, and seeking women's own views about how services can be improved in their local area.

The second thorny issue is how to prevent relapse to smoking during pregnancy and in the post-partum period. We have no reliable data in the United Kingdom on relapse amongst pregnant women who have accessed NHS stop smoking services. However, we do have evidence from other UK studies and international research which suggests that rates of relapse remain high. Up to $60 \%$ of women who stop smoking at some point during pregnancy relapse within six months of the birth and up to $80 \%$ return to smoking within twelve months (Floyd et al., 1993, Fingerhut et al., 1990). Little progress has been made in designing effective relapse prevention strategies for pregnant women although research which examines the factors that predict or protect against relapse is developing and may provide the basis for future intervention development.

\section{Conclusion}

The benefits of smoking cessation during pregnancy are clear for both mothers and children, but significant barriers remain in supporting women to quit. Some of these barriers relate to women's personal and social circumstances and their views about cessation and accessing services. Other barriers relate to significant gaps in the identification and referral of pregnant smokers to services that are, in the United Kingdom at least, available to support women to quit and have been found to be effective in the short term. Clearly more needs to be done to improve the quality of data on smoking during pregnancy, to improve the reach of specialist services and to inform women about these services and make them as accessible and available as possible. Unless pregnant smokers can be accurately identified, automatically referred and then encouraged to engage with treatment, then the contribution of NHS stop smoking services to reducing rates of smoking during pregnancy is likely to remain modest at best.

\section{Acknowledgments}

Some of the findings outlined in this article are from a study funded by Health Scotland. The views expressed are those of the author and not necessarily those of Health Scotland. The author is grateful to her colleagues David Tappin, Susan Macaskill and Douglas Eadie who contributed to the Health Scotland study. The author is part of the UK Centre for Tobacco Control Studies, a UKCRC Public Health Research Centre of Excellence. 
Funding from British Heart Foundation, Cancer Research UK, Economic and Social Research Council, Medical Research Council, and the Department of Health, under the auspices of the UK Clinical Research Collaboration, is gratefully acknowledged.

\section{References}

ASH. (2008) Beyond smoking kills. Available at http://ash. org.uk/ash_3xe9h0zo.htm

Bauld, L., Wilson, M., Kearns, A., \& Reid, M. (2007). Exploring reductions in smoking in pregnancy in Glasgow. Glasgow Centre for Population Health, Glasgow, June 2007.

Batstra, L., Hadders-Algra, M., \& Neeleman, J. (2003). Effect of antenatal exposure to maternal smoking on behavioural problems and academic achievement in childhood. Early Human Development, 75, 21-33.

Bell, K., Bauld, L., McCullough, L., Greaves, L., \& Jategaonkar, K., and DeVries, K. (2007). The effectiveness of national health service intensive treatments for smoking cessation in England: A systematic review. London: National Institute for Clinical Excellence. Available at http://www.nice.org.uk/page.aspx?o=404427

British Medical Association (2007) Breaking the cycle of childrens' exposure to tobacco smoke. BMA Board of Science, London.

Charlton, A. (1996). Children and smoking: the family circle. British Medical Bulletin, 52, 90-107.

Fingerhut, L., Kleinman, J., \& Kendrick, J. (1990) Smoking before, during, and after pregnancy. American Journal of Public Health, 80, 541-544.

Floyd, R.L., Rimmer, B., Giovino, G. et al. (1993) A review of smoking in pregnancy: Effects on pregnancy outcomes and cessation efforts. Annual Review of Public Health, 14, 379-411.

Ford, R., Tappin, D., Schulter, P. et al. (1997) Smoking during pregnancy: How reliable are maternal self-reports in New Zealand? Journal of Epidemiology and Community Health, 51, 246-251.

Greaves, L., Cormier, R., Devries, K. et al. (2003) Expecting to quit: a best practices review of smoking cessation interventions for pregnant and postpartum girls and women.
Vancouver: British Columbia Centre of Excellence for Women's Health.

Information and Statistics Division. (2008) NHS Smoking cessation service statistics (Scotland), 1st January to 31st December 2007. Available at http://www.isdscotland.org/ isd/5459.html

Information Centre. (2007). Infant Feeding Survey: 2005 Results. Available at http://www.ic.nhs.uk/statistics-anddata-collections/health-and-lifestyles-related-surveys/infantfeeding-survey

Lawrence, T., Aveyard, P., Cheng, K. et al. (2005) Does stagebased smoking cessation advice in pregnancy result in long-term quitters? 18-month postpartum follow-up of a randomized controlled trial. Addiction 100, 107-116.

Lowry, R.J., Hardy, S., Jordan, C. and Wayman, G. (2004). Using social marketing to increase recruitment of pregnant smokers to smoking cessation service: a success story. Public Health, 118, 239-243.

Macaskill, S., Bauld, L., Eadie, D., \& Tappin, D. (2008) Smoking cessation support in pregnancy in Scotland, Health Scotland, Glasgow. Available at http://www.healthscotland. com/documents/2665.aspx

Naughton, F. (2008, June). Why do so few pregnant smokers seek help from the Stop Smoking Services? Paper presented at the UK National Smoking Cessation Conference, Birmingham.

O'Gorman, C. (2008) We need better data on smoking in pregnancy. British Medical Journal, 336, 330.

Royal College of Physicians. (1992). Smoking and the Young. A report of a working party of the Royal College of Physicians. London: Author.

Usmani, Z., Craig, P., Shipton, D., \& Tappin, D. (2008) Comparison of $\mathrm{CO}$ breath testing and women's self-reporting of smoking behaviour for identifying smoking during pregnancy. Substance Abuse Treatment Prevention and Policy, 3, 4.

Ussher, M., Etter, J.F., \& West, R. (2006) Perceived barriers to and benefits of attending a stop smoking course during pregnancy. Patient Education \& Counselling, 61, 467-472. 